\title{
TETRAHEDRA ASSOCIATED WITH CANONICAL EXPANSIONS FOR A CURVED SURFACE*
}

\author{
BY P. O. BELL
}

A number of different canonical developments for the equation of a curved surface have been obtained by investigators in the field of projective differential geometry. These developments $\dagger$ are simplest in form when the vertices of associated tetrahedra are located on a certain quadric, known as the canonical quadric of Wilczynski. $\ddagger$ The geometrical location of this quadric was accomplished by Wilczynski by a very complicated method. Bompiani§ has offered a distinctly different definition, and Stouffer $\|$, using the general methods of Wilczynski, has found a rather simple method of locating the quadric.

The canonical quadric is actually useful in locating only one of the four vertices of the tetrahedron. It is the purpose of this paper to locate the fourth vertex for the whole series of expansions by rather elementary methods and without the introduction of Wilczynski's quadric. As a matter of fact, the quadric is located as soon as any one of these fourth vertices is determined.

We shall suppose that the asymptotic net is parametric, and take the fundamental differential equations in the form

$$
y_{u u}+2 b y_{v}+f y=0, \quad y_{v v}+2 a^{\prime} y_{u}+g y=0 .
$$

Using the notation introduced in the celebrated memoir by Green we shall put

$$
\rho=y_{u}-\beta y, \quad \sigma=y_{v}-\alpha y,
$$

where $\alpha$ and $\beta$ are functions of $u$ and $v$ which may be assigned as desired. If the point $y$ on the surface and the corresponding points $\rho$ and $\sigma$ are chosen as three vertices of the tetrahedron

* Presented to the Society, April 6, 1935.

† Green, Transactions of this Society, vol. 20 (1919), pp. 79-153.

$\ddagger$ Wilczynski, Transactions of this Society, vol. 9 (1908), pp. 79-120.

\& Bompiani, Rendiconti Accademia Lincei, (6), vol. 6 (1927), pp. 187-190, and Mathematische Zeitschrift, vol. 29 (1929), pp. 678-683.

\| Stouffer, Proceedings of the National Academy of Sciences, (18), vol. 3 (1932), pp. 252-255. 
of reference, the associated development for the equation of the surface takes its simplest form if the fourth vertex $\tau$ is given by

$$
\tau=y_{u v}-\alpha y_{u}-\beta y_{v}+\alpha \beta y .
$$

Under these conditions the point $\tau$ is on the quadric of Wilczynski for all values of $\alpha$ and $\beta$. Moreover, the line $l^{\prime}$ joining $y$ and $\tau$ is the reciprocal with respect to any quadric of Darboux of the line $l$ joining $\rho$ and $\sigma$. Furthermore, the tangent plane at $\rho$ to the ruled surface $R^{(u)}$ generated by the line joining $y$ and $\rho$ as $y$ moves along $C_{u},(v=$ const. curve), and the tangent plane at $\sigma$ to the ruled surface $R^{(v)}$ generated by the line joining $y$ and $\sigma$ as $y$ moves along $C_{v},(u=$ const. curve $)$ intersect in the line $l^{\prime}$. If $\beta=a_{u}^{\prime} /\left(2 a^{\prime}\right), \alpha=b_{v} /(2 b)$, the lines $l$ and $l^{\prime}$ are the directrices of Wilczynski of the first and second kind, respectively, and if $\beta=-b_{u} /(4 b), \alpha=-a_{v}^{\prime} /\left(4 a^{\prime}\right)$, they are the canonical edges of Green of the first and second kind, respectively. These lines and consequently the associated points $\rho$ and $\sigma$ are not difficult to locate geometrically. The above stated facts are well known and may be found, for example, in the memoir by Green.*

Let us now consider the set of points

$\rho_{i}=y_{u}-\beta_{i} y, \sigma_{j}=y_{v}-\alpha_{j} y, \tau_{i j}=y_{u v}-\alpha_{j} y_{u}-\beta_{i} y_{v}+\alpha_{j} \beta_{i} y$.

The reciprocal lines $l$ and $l^{\prime}$ determined by $\rho_{i}$ and $\sigma_{j}$ we shall denote by $l_{i j}$ and $l_{i j}$. In particular we shall denote the directrices of Wilczynski by $l_{11}$ and $l_{11}^{\prime}$ with the corresponding points denoted by

$\rho_{1}=y_{u}-\beta_{1} y, \sigma_{1}=y_{v}-\alpha_{1} y, \tau_{11}=y_{u v}-\alpha_{1} y_{u}-\beta_{1} y_{v}+\alpha_{1} \beta_{1} y$.

Similarly, the canonical edges of Green will be denoted by $l_{22}$ and $l_{22}^{\prime}$ with the subscript 2 attached to the corresponding points $\rho, \sigma$, and $\tau$.

Two points $\rho_{p}, \rho_{q}$ and two points $\sigma_{r}, \sigma_{s}$ determine four lines $l_{p r}, l_{q s}, l_{p s}, l_{q r}$. The lines $l_{p r}$ and $l_{q s}$ intersect in a point $l_{p r} l_{q s}$ which is easily found to be denoted by

$$
\left(\alpha_{r}-\alpha_{s}\right) y_{u}+\left(\beta_{q}-\beta_{p}\right) y_{v}+\left(\alpha_{s} \beta_{p}-\alpha_{r} \beta_{q}\right) y .
$$

This point is identical with $\tau_{p s}-\tau_{q r}$. Therefore, the points $l_{p r} l_{q s}, \tau_{p s}, \tau_{q r}$ are collinear. Similarly, the points $l_{q r} l_{p s}, \tau_{q s}, \tau_{p r}$

* Green, loc. cit. 
may be shown to be collinear. Moreover, the above proof holds when $\rho_{p} \equiv \rho_{q}$, or when $\sigma_{r} \equiv \sigma_{s}$. We therefore have the following theorem.

TheOREM. The points $\tau_{p s}, \tau_{q r}$, and $l_{p r} l_{q s}$ are collinear.

Now the points $\tau_{12}$ and $\tau_{21}$ may be located geometrically, as follows. The points $\rho$ and $\sigma$ for given functions $\alpha$ and $\beta$ generate two surfaces, $s_{\rho}$ and $s_{\sigma}$. The tangent at $\rho$ to the curve $u=$ const. on $s_{\rho}$ and the tangent at $\sigma$ to the curve $v=$ const. on $S_{\sigma}$ intersect the line $l^{\prime}$ in the points $\tau-\beta_{v} y$ and $\tau-\alpha_{u} y$, respectively. The harmonic conjugate of $y$ with respect to these two points is the point $\tau-\left(\alpha_{u}+\beta_{v}\right) y / 2$. The harmonic conjugate of the point $\tau-\beta_{v} y$ with respect to the two points $\tau-\alpha_{u} y$ and $\tau-\left(\alpha_{u}+\beta_{v}\right) y / 2$ is the point $\tau-\left(2 \alpha_{u}+\beta_{v}\right) y / 3$. This is the point $\tau$ if $\alpha$ and $\beta$ are chosen to satisfy the condition

$$
2 \alpha_{u}+\beta_{v} \equiv 0 .
$$

This condition is satisfied by the selection

$$
\alpha=-\frac{a_{v}^{\prime}}{4 a^{\prime}}, \quad \beta=\frac{a_{u}^{\prime}}{2 a^{\prime}} .
$$

But for this selection $\rho \equiv \rho_{1}$ and $\rho \equiv \sigma_{2}$. The point $\tau$ for this selection of $\alpha$ and $\beta$ is therefore the point $\tau_{12}$. The geometric definition of the point $\tau_{21}$ is obtained from the definition of $\tau_{12}$ by interchanging $u$ and $v, \rho$ and $\sigma, \alpha$ and $\beta$, and replacing $a^{\prime}$ by $b$.

It follows from the above theorem that the points $l_{12} l_{11}, \tau_{11}$, and $\tau_{12}$ are collinear; also, $l_{11} l_{21}, \tau_{11}$, and $\tau_{21}$ are collinear. Thus $\tau_{11}$ is the intersection of the line determined by $l_{12} l_{11}$ and $\tau_{12}$ and the line determined by $l_{11} l_{21}$ and $\tau_{21}$, both of which are geometrically located. The point $\tau_{22}$ may be located similarly.

We are now in a position to locate any point $\tau_{r s}$. The points $l_{1 s} l_{r 1}, \tau_{11}$ and $\tau_{r s}$ are collinear, as are also the points $l_{2 s} l_{r 2}, \tau_{22}$, and $\tau_{r s}$. But each of these lines passes through two known points and their intersection determines $\tau_{r s}$. Therefore, upon specifying the locations of the points $\rho_{r}$ and $\sigma_{s}$, the geometric definition of the point $\tau_{r s}$ is complete.

Stanford University 